\title{
Parásitos metazoos de Anas georgica Gmelin, 1789 (Aves: Anseriformes) en Chile central: especificidad, prevalencia y variaciones entre localidades
}

\author{
Host specificity, prevalence and between-sites variation in metazoan parasites of Anas \\ georgica Gmelin, 1789 (Aves: Anseriformes) in Chile
}

\author{
ANA HINOJOSA-SÁEZ ${ }^{1,2, *}$, DANIEL GONZÁLEZ-ACUÑA ${ }^{3} \&$ MARIO GEORGE-NASCIMENTO ${ }^{4}$

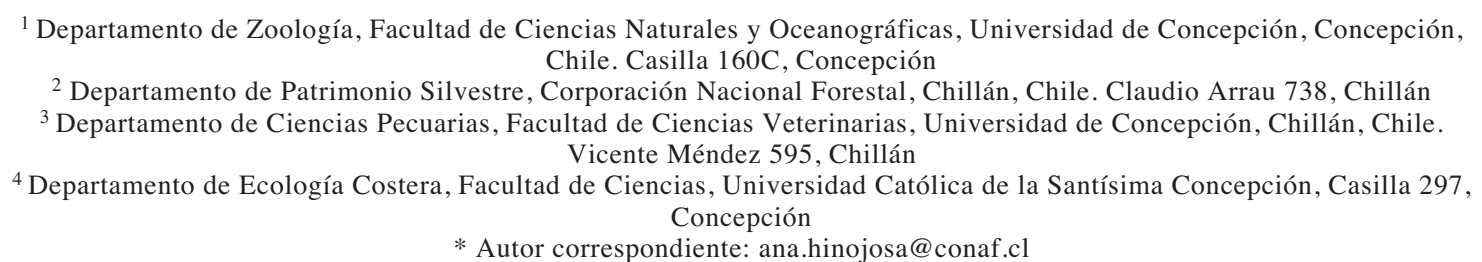

\section{RESUMEN}

Se estudiaron los parásitos metazoos en 65 ejemplares del pato jergón grande Anas georgica Gmelin, 1789 (Aves: Anseriformes), capturados entre los meses de mayo y julio de 2004, en seis localidades de la zona centro-sur de Chile. Se evaluó si existía correlación entre el valor del índice de especificidad STD (Statistical Taxonomic Distinctiveness), para cada taxón determinado a nivel de especie, con sus respectivas prevalencias e intensidades. Además, se evaluó la significancia estadística de las variaciones entre localidades en la prevalencia e intensidad de ectoparásitos y endoparásitos, en la abundancia total de parásitos y en la riqueza de las infracomunidades. Finalmente, se evaluó si el peso corporal y el sexo de los hospedadores eran relevantes para entender las variaciones de la abundancia de cada taxon, la abundancia total y la riqueza en las infracomunidades. En el $81.5 \%$ de los ejemplares examinados se encontraron 1,653 parásitos, pertenecientes a un total de 11 taxa, a saber, los ectoparásitos del orden Phthiraptera Anaticola crassicornis (Scopoli, 1763), Anatoecus icterodes (Nitzsch, 1818) y Trinoton querquedulae (Linneus, 1758), y los endoparásitos Digenea Australapatemon burti (Miller, 1923) Dubois, 1968, Notocotylus imbricatus (Loss, 1893), Paramonostomum pseudoalveatum Price, 1931, Echinostoma sp. Rudolphi, 1809, Echinoparyphium sp. Dietz, 1909, el Nematoda Porrocaecum sp. Railliet \& Henry, 1912 y los Cestoda Cloacotaenia megalops (Nitzsch in Creplin, 1829) Wolfhuegel, 1938 y Fuhrmanacanthus propeteres (Fuhrmann, 1907) Spasskii, 1966. La correlación entre el índice de especificidad STD y la prevalencia de los taxa parasitarios resultó ser negativa y significativa, no así la correlación con la intensidad. No se encontraron diferencias significativas en la abundancia o riqueza de los parásitos entre sexos de las aves, pero sí en la riqueza de especies y en la abundancia total entre sitios. Con excepción de los Digenea y el piojo A. icterodes, todos los demás parásitos constituyen primer registro en Chile. Para los parásitos de ciclo heteroxeno, no se conocen sus ciclos vitales ni las especies que actuarían como hospedadores intermediarios, en Chile.

Palabras clave: Anas georgica, índice de especificidad, intensidad, parásitos, prevalencia.

\section{ABSTRACT}

Host specificity, prevalence and between-sites variation in metazoan parasites of 65 Anas georgica Gmelin, 1789 (Aves: Anseriformes) specimens were assessed at six localities sampled between May and July 2004 in the central-south zone of Chile. The correlation between the Statistical Taxonomic Distinctiveness index (STD) with the prevalence and intensity in each parasite taxon was assessed in those taxa with determination attained at the species level. Furthermore, the statistical significance of between-sites variation in abundance and prevalence of ectoparasites and endoparasites were assessed as well as those in total abundance and 
taxonomic richnes of infracommunities. Finally, statistical tests allowed assessing whether host body weight and sex were relevant sources of variation in abundance and richness. Most ducks (81.5\%) harbored parasites and from which 1,653 individuals belonging to 11 taxa were collected. They were, among ectoparasites, the Phthiraptera Anaticola crassicornis (Scopoli, 1763), Anatoecus icterodes (Nitzsch, 1818) and Trinoton querquedulae (Linneus, 1758). Among the helminths we found the Digenea Australapatemon burti (Miller, 1923) Dubois, 1968, Notocotylus imbricatus (Loss, 1893), Paramonostomum pseudoalveatum Price, 1931, and an unidentified species of Echinostoma sp. Rudolphi, 1809, Echinoparyphium Dietz, 1909; the Nematoda Porrocaecum sp. Railliet \& Henry, 1912, and the Cestoda Cloacotaenia megalops (Nitzsch in Creplin, 1829) Wolfhuegel, 1938 and Fuhrmanacanthus propeteres (Fuhrmann, 1907) Spasskii, 1966. All parasites except for Digenea and A. icterodes are recorded for the first time in Chile. There was a significantly negative correlation between STD index and the prevalence while there was no significant correlation between hostspecificity and intensity. There were no between-sex differences in abundance or richness, but these variables showed differences between sites. There is no information on life cycles of the collected parasites or intermediate hosts involved in their transmisssion in Chile.

Key words: Anas georgica, host specificity index, intensity, parasites, prevalence.

\section{INTRODUCCIÓN}

Las especies de parásitos que ocupan diferentes hospedadores en forma exitosa, deben tolerar las diferencias fisiológicas, morfológicas y/o de sus sistemas inmunes. Esta capacidad tendría un costo de inversión energética por parte del parásito, lo que podría ir en desmedro de otras actividades importantes como la reproducción (Poulin, 1999). Por lo expuesto, se ha propuesto una correlación negativa entre la especificidad de un parásito, calculado a través del índice STD, y su prevalencia e intensidad (Poulin 1998, 1999, Simková et al., 2002).

La especificidad que un parásito exhibe por sus diferentes hospedadores se ha relacionado en la mayoría de los casos solo con su rango de hospedadores, es decir, con el número de especies hospederas de un parásito en un determinado estado de desarrollo (Poulin \& Keeney, 2008). Sin embargo, los parásitos que poseen igual rango de hospedadores, no necesariamente son igualmente específicos, ya que la distancia taxonómica de los hospedadores puede ser radicalmente distinta. En este contexto, el índice STD (Statistical Taxonomic Distinctiveness), desarrollado por Clarke \& Warwick $(1999,2001)$, y modificado por Poulin \& Moulliot (2003) para ser aplicado en parasitología, establece que, entre más distantes taxonómicamente sean los hospedadores, mayor será el valor de este índice, y por lo tanto, menor debería ser la especificidad del parásito. Las ventajas de este índice son que es fácilmente calculable, independiente del esfuerzo de estudio o tamaño de muestra, y que tiene la facultad de discernir entre parásitos con similar rango de hospedadores. Este índice ha sido aplicado en parásitos de peces marinos (Luque et al. 2004) y en parásitos de aves, incluidos Anseriformes (Poulin \& Mouillot 2004).

Los registros parasitarios en hospedadores que habitan sistemas de agua dulce en Chile se han enfocado principalmente en hospedadores vertebrados como peces (18 especies), anfibios (11 especies) y aves (11 especies) (Olmos \& Muñoz, 2006). En hospedadores invertebrados existen solo dos trabajos, uno en nemátodos del crustáceo decápodo Aeglea denticulada Nicolet, 1842 (Crustacea: Decapoda) (Torres \& Jara, 1986) y el otro realizado en digeneos del molusco gastrópodo Chilina dombeyana (Brugiere, 1789) (Mollusca: Gastropoda) (Olmos \& George-Nascimento, 1997). Los estudios en parasitismo de aves del orden Anseriformes solo contemplan registros en Chloephaga picta (Gmelin, 1789) en la Región de Magallanes (González et al., 2005), y en Cygnus melancoryphus Molina, 1782 en Valdivia (Schlatter et al., 1991). En Chile, solo existe el registro de las especies Australapatemon burti (Millar, 1923) y Paramonostomum pseudoalveatum Price, 1931 (Platyhelminthes: Digenea) (Drago et al., 2007). En Argentina, se cuenta con los registros de Anaticola crassicornis (Scopoli, 1763), Anatoecus icterodes (Nitzsch, 1818) y Trinoton querquedulae (Linneus, 1758) (Cicchino \& Del Castro, 1998, Price et al., 2003) (Insecta: Phthiraptera), y en Brasil, se registró Cloacotaenia megalops (Platyhelminthes: Cestoda) (Nitzsch in Creplin, 1829) Wolffhügel, 1938 (Muniz-Pereira \& Amato, 1998). 
El pato jergón grande Anas georgica Gmelin, 1789 se distribuye en gran parte de América del Sur, desde Colombia hasta la Patagonia chileno-argentina, incluyendo las islas australes del canal Beagle y en forma ocasional en las Islas Shetland del Sur y la Antártica (Kolbe, 1999). Su hábitat son orillas de ríos, lagos, lagunas y todo tipo de cuerpos de agua dulce hasta los 1,000 msnm (Housse, 1945) y ocasionalmente, se encuentra en campos inundados y ambientes salinos (Martínez \& González, 2004). En Chile está descrito como uno de los anátidos más comunes (Madge \& Burn 2000, Martínez \& González, 2004), con dos períodos de reproducción, uno en agosto y otro entre enero y febrero (Madge \& Burn, 2000), vive en pareja durante el período de cría y en bandadas durante los meses invernales. Es descrita como un ave omnívora, encontrándose en su dieta semillas, hierbas, moluscos, insectos, pequeños peces y anfibios (Kolbe, 1999, Figueroa et al., 2001).

En este trabajo se estudia la composición de la fauna parasitaria de A. georgica, sus variaciones entre las localidades de captura, y se analiza si el peso corporal y el sexo de los hospedadores son relevantes para entender las variaciones de la abundancia de cada taxon, la abundancia total y la riqueza de especies en las infracomunidades. Finalmente, se evalúa la correlación de la especificidad parasitaria con la prevalencia e intensidad parasitaria, luego de obtener su rango de hospedadores mediante una exhaustiva revisión de la literatura.

\section{MÉTODOS}

Entre el 1 abril y el 31 de julio de 2004, período permitido de caza (SAG 2001), se capturaron con arma de fuego un total de 65 ejemplares de A. georgica en 6 localidades de las regiones del Maule y Biobío, Chile (Tabla 1). Cada ave fue almacenada en bolsas individuales, debidamente selladas y mantenidas a $4{ }^{\circ} \mathrm{C}$ hasta su inspección. Todas fueron pesadas con una balanza de $10 \mathrm{~g}$ de precisión, sin embargo se consideraron solo 55 datos por falta de integridad física de las restantes aves. Se registró el sexo de cada ejemplar y la localidad de muestreo. Los ectoparásitos se obtuvieron mediante búsqueda intensiva en el plumaje sobre una superficie blanca durante 10 a $15 \mathrm{~min}$, la que fue revisada bajo lupa con una magnificación de $20 x$. Cada ectoparásito fue montado siguiendo los procedimientos descritos por Palma (1978) y Price et al. (2003), y luego, determinados taxonómicamente bajo microscopio óptico según Kéler $(1957,1960)$, Clay (1963), Price \& Beer (1963), Eichler \& Vasjukova (1980) y Tendeiro \& Mendes (1994). Luego, cada ejemplar fue sometido a una necropsia parasitaria de Whitlock modificada (Cattan \& Tagle, 1974) en búsqueda de helmintos gastrointestinales. La determinación taxonómica de los helmintos se basó en las claves de Skrjabin (1964) y Yamaguti (1958) para trematodos, y para cestodos se siguió a Yamaguti (1959),

TABLA 1

Número de ejemplares de Anas georgica muestreados en cinco localidades de Chile central (ordenadas de norte a sur). Se indica número de machos, hembras y el peso promedio ( $\mathrm{g}$ ) de las aves para cada localidad.

Number of Anas georgica sampled at six localities in central Chile (from north to south). Number of males, females and mean body mass $(\mathrm{g})$ of birds for each locality are shown.

\begin{tabular}{|c|c|c|c|c|}
\hline Localidad (coordenadas) & $\mathrm{N}$ machos & $\mathrm{N}$ hembras & Peso machos & Peso hembras \\
\hline Quella $\left(36^{\circ} 03^{\prime} \mathrm{S}-72^{\circ} 05^{\prime} \mathrm{O}\right)$ & 9 & 5 & 730.3 & 672.6 \\
\hline Parral (36 $\left.06^{\prime} \mathrm{S}-71^{\circ} 50^{\prime} \mathrm{O}\right)$ & 12 & 7 & $740.8(n=7)$ & $662.0(\mathrm{n}=2)$ \\
\hline Niquén $\left(36^{\circ} 13^{\prime} \mathrm{S}-72^{\circ} 03^{\prime} \mathrm{O}\right)$ & 2 & 4 & 780.0 & 602.5 \\
\hline San Nicolás $\left(36^{\circ} 29^{\prime} \mathrm{S}-72^{\circ} 13^{\prime} \mathrm{O}\right)$ & 6 & 2 & 746.6 & 720.0 \\
\hline Aeródromo $\left(36^{\circ} 34^{\prime} \mathrm{S}-72^{\circ} 03^{\prime} \mathrm{O}\right)$ & 4 & 5 & 537.5 & 542.0 \\
\hline Tubul $\left(37^{\circ} 14^{\prime} \mathrm{S}-73^{\circ} 25^{\prime} \mathrm{O}\right)$ & 8 & 1 & 634.6 & 677.0 \\
\hline Total & 41 & 24 & 594.9 & 646.0 \\
\hline
\end{tabular}


Schmidt (1970), Schmidt \& Roberts (1984), Khalil \& Jones (1994) y Yamaguti (1961). Finalmente, para nemátodos se utilizó la clave de Anderson et al. (1974).

El índice de especificidad (STD) fue calculado para cada taxón parásito identificado a nivel específico a través de la fórmula dada por Poulin \& Mouillot (2003) que usa la clasificación Linneana, siendo el valor máximo cinco (cuando los hospedadores corresponden a diferentes clases) y el valor mínimo uno (cuando los hospedadores son congenéricos). Para el cálculo de este índice fue necesario elaborar para cada parásito, un rango de hospedadores, basado en los antecedentes bibliográficos. Además, se calculó la varianza de este índice ya que permite discernir entre parásitos que obtengan un valor de STD similar (Poulin \& Mouillot 2003).

La prevalencia e intensidad de infección de cada taxón parasitario fue calculada siguiendo a Bush et al. (1997). Además, se registró para cada hospedador, el número total de taxa parasitarios (riqueza taxonómica) y la abundancia total. En el análisis estadístico de los datos se utilizó el coeficiente de correlación de Spearman para evaluar la asociación entre el peso corporal de cada ave con la intensidad de cada taxón parasitario, la riqueza taxonómica y la abundancia total. El mismo coeficiente fue empleado para el análisis de la asociación entre la especificidad parasitaria y la prevalencia e intensidad de infección (solo para aquellos taxa en que se alcanzó el nivel específico en su determinación taxonómica). Para evaluar la significancia estadística de las variaciones entre sexos de la intensidad de cada taxón, de la riqueza de especies y de la abundancia total de parásitos, se utilizaron pruebas "U" de Mann-Whitney. Por último, las variaciones de las mismas variables entre localidades de muestreo se evaluaron mediante la prueba de Kruskal-Wallis (Zar 1984).

\section{RESULTADOS}

El peso promedio de las aves muestreadas fue de $671.9 \mathrm{~g}(\mathrm{DE}=131.2$, rango $=400-900, \mathrm{n}=$ 55, Tabla 1). El $81.5 \%$ de las aves albergaban al menos un taxón parasitario, recolectándose 1,653 individuos pertenecientes a un total de
11 taxa, a saber, ectoparásitos del orden Phthiraptera, Anaticola crassicornis (Scopoli 1763), Anatoecus icterodes (Nitzsch, 1818) y Trinoton querquedulae (Linneus, 1758), los endoparásitos Digenea, Australapatemon burti (Miller, 1923) Dubois, 1968, Notocotylus imbricatus (Loss, 1893), Paramonostomum pseudoalveatum Price, 1931, Echinostoma sp. Rudolphi, 1809, Echinoparyphium sp. Dietz, 1909, el Nematoda Porrocaecum sp. Railliet \& Henry, 1912 y los Cestoda Cloacotaenia megalops (Nitzsch in Creplin, 1829) Wolfhuegel, 1938 y Fuhrmanacanthus propeteres (Fuhrmann, 1907) Spasskii, 1966. La prevalencia, intensidad, rango de hospedadores e índice de especificidad STD de las especies encontradas se detallan en la Tabla 2.

La correlación entre el índice de especificidad STD y la prevalencia de los taxa que fueron determinados a nivel específico resultó ser negativa y significativa $\left(\mathrm{r}_{\mathrm{s}}=-0.73 ; \mathrm{P}\right.$ $<0.05 ; \mathrm{n}=8)$, sin embargo esto no fue así con la intensidad $\left(\mathrm{r}_{\mathrm{s}}=0.04 ; 0.99>\mathrm{P}>0.90 ; \mathrm{n}=8\right)$.

Ningún taxón mostró asociación significativa entre el peso corporal de los hospedadores y su abundancia $(\mathrm{P}>0.05$ en todos los casos). En forma similar, no se encontró correlación significativa entre dicha variable y la riqueza taxonómica $\left(\mathrm{r}_{\mathrm{s}}=0.13\right.$; $0.30<\mathrm{P}<0.40 ; \mathrm{n}=55)$ o la abundancia total $\left(\mathrm{r}_{\mathrm{s}}=0.07 ; 0.50<\mathrm{P}<0.60 ; \mathrm{n}=55\right)$. No se encontraron diferencias significativas en la abundancia o riqueza de los parásitos entre los sexos de las aves (Prueba "U" de MannWhitney; P > 0.05).

Se encontraron diferencias significativas entre localidades en la riqueza de especies ( $F$ $\left.{ }_{(5.59)}=9.33 ; \mathrm{P}<0.001\right)$ y en la abundancia total de parásitos $\left(\mathrm{F}_{(5,59)}=5.34 ; \mathrm{P}<0.001\right.$, Tabla 3). Los ejemplares muestreados en Tubul, Quella y Parral tenían más taxa parasitarios que los muestreados en San Nicolás y el aeródromo de Chillán. Las diferencias en abundancia total de parásitos entre localidades radican en que los patos muestreados en Tubul tenían mayor abundancia que los muestreados en San Nicolás y el aeródromo de Chillán. El único parásito que mostró diferencias de abundancia entre localidades fue $P$. pseudoalveatum, el cual tenía mayor abundancia en Tubul que en Parral. 


\section{TABLA 2}

Parásitos encontrados en Anas georgica en Chile central, se indica su Prevalencia $(\mathrm{P}, \mathrm{n}=65)$, Intensidad (I), Rango de Hospedadores (RH), Índice de Especificidad (STD), Varianza (VarSTD) y coeficiente de correlación de Spearman $(n=55)$ entre la abundancia de cada uno de los taxa parasitarios y el peso corporal de las aves.

Parasite taxa found in Anas georgica in central Chile, prevalence $(\mathrm{P}, \mathrm{n}=65)$, Intensity $(\mathrm{I})$, host range $(\mathrm{RH})$, specificity index (STD), variance (varSTD) and Spearman's correlation coefficient $(\mathrm{n}=55)$ between the abundance of each taxon and host body mass.

\begin{tabular}{lcccccc}
\hline Parásitos & $\mathrm{P}(\%)$ & $\mathrm{I}$ & $\mathrm{RH}$ & $\mathrm{STD}$ & VarSTD & $\mathrm{r}_{\mathrm{s}}$ \\
\hline Phthiraptera & & & & & & \\
Anaticola crassicornis & 55.4 & 3.4 & 39 & 1.2 & 0.08 & 0.16 \\
Anatoecus icterodes & 26.1 & 1.6 & 89 & 1.8 & 0.06 & -0.03 \\
Trinoton querquedulae & 36.9 & 1.6 & 87 & 1.8 & 0.09 & 0.11 \\
Digenea & & & & & & \\
Australapatemon burti & 27.7 & 8.7 & 7 & 1.3 & 0.13 & 0.20 \\
Paramonostomum pseudoalveatum & 9.2 & 136.7 & 15 & 2.2 & 0.64 & -0.17 \\
Notocotylus imbricatus & 1.5 & 1.0 & 16 & 2.4 & 1.08 & 0.04 \\
Echinostoma sp. & 3.1 & 1.0 & & & & \\
Echinoparyphium sp. & 1.5 & 3.0 & & & & \\
Nematoda & & & & & & \\
Porrocaecum sp. & 3.1 & 2.5 & & & & \\
Cestoda & & & & & & \\
Cloacotaenia megalops & 6.1 & 6.7 & 63 & 2.0 & 0.43 & -0.10 \\
Fuhrmanacanthus propeteres & 46.1 & 15.4 & 7 & 2.0 & 0.00 & 0.11 \\
\hline
\end{tabular}

TABLA 3

Localidad de muestreo (ordenadas de norte a sur), número de ejemplares de Anas georgica muestreados (n), riqueza, abundancia total de parásitos $\log _{10}(\mathrm{x}+1)$. Desviación estándar entre paréntesis.

Sampling localities (from north to south), number of Anas georgica sampled (n), parasite richness, total parasite abundance $\log _{10}(\mathrm{x}+1)$. Standard deviation in parenthesis.

\begin{tabular}{lccc}
\hline Localidad & $\mathrm{n}$ & Riqueza & Abundancia total \\
\hline Quella & 14 & $2.86(1.23)$ & $0.939(0.438)$ \\
Parral & 19 & $2.58(1.17)$ & $0.992(0.359)$ \\
Niquén & 6 & $1.50(1.05)$ & $0.918(0.760)$ \\
San Nicolás & 8 & $0.87(0.83)$ & $0.682(0.652)$ \\
Aeródromo & 9 & $0.55(0.88)$ & $0.379(0.579)$ \\
Tubul & 9 & $2.89(0.93)$ & $1.605(0.611)$ \\
Total & 65 & $2.09(1.38)$ & $0.935(0.609)$ \\
\hline
\end{tabular}

\section{DISCUSIÓN}

No es posible afirmar tajantemente que las adaptaciones morfológicas de los Phthiraptera estén relacionadas directamente con los hábitos de su hospedador (Cicchino \& Del Castro, 1998), ya que muchos otros factores, tales como la distribución discontinua de géneros y especies, la evolución convergente, infestaciones secundarias y errores humanos en la determinación taxonómica, pueden provocar incertidumbres en las posibles explicaciones al respecto (Marshall, 1981). En otros ectoparásitos de vertebrados, específicamente en pulgas de roedores, se describe una mayor prevalencia en parásitos que comparten hospedadores cercanos taxonómicamente (Krasnov et al., 2004), lo que concuerda con lo encontrado en este trabajo, ya que $A$. crassicornis, la especie con el menor índice 
STD (lo que indica una estrecha relación entre sus hospedadores), registró la mayor prevalencia, en tanto que $A$. icterodes y $T$. querquedulae presentaron valores de especificidad y varianzas levemente diferentes, y a la vez sus prevalencias no presentaron diferencias significativas.

Entre las aves, el orden Anseriformes posee el mayor número de registros de Phthiraptera (Price et al., 2003), lo que explica el amplio rango de hospedadores en las tres especies de Phthiraptera (entre 39 y 89 especies, ver Tabla 2). En este sentido, si se considera solo el rango de hospedadores para establecer la especificidad de cada parásito, concluiríamos que las tres son especies generalistas, pero los valores del índice STD (entre uno y dos) evidencian lo contrario, debido a que sus hospedadores son confamiliares, por lo que estos parásitos, al igual que sus hospedadores, deben compartir una estrecha historia evolutiva (Poulin, 1999).

La facilidad con que un ectoparásito se mueve fuera de su hospedador es un factor que se ha asociado a las variaciones de especificidad. Por esto, las especies del orden Phthiraptera han sido usadas para realizar estudios de coevolución en sistemas hospedador-parásito, ya que poseen una escasa capacidad de desplazamiento y por lo tanto una estrecha relación con sus hospedadores. (Hafner et al., 1994, Price et al., 2003). Las especies del suborden Ischnocera son más específicas aún, debido a que se alimentan solo de plumas, son poco activas, y muestran predilección por ciertos sitios corporales sobre el ave. En contraste, las especies del suborden Amblycera se alimentan tanto de plumas como de sangre, son más activas y menos específicas en cuanto al sitio del cuerpo del ave que ocupan (Marshall, 1981). Esto concuerda con los resultados del presente estudio, ya que la especie más específica fue A. crassicornis, la cual pertenece al suborden Ischnocera, a diferencia de las otras dos, que pertenecen al suborden Amblycera (Tabla 2).

Las diferencias en la conducta de los hospedadores pueden llevar a diferentes niveles de infestación. Por ejemplo, los hospedadores de hábitos coloniales tendrían mayor número de especies ectoparásitas que las especies solitarias (Marshall, 1981). Esto podría explicar la presencia de tres especies de Phthiraptera en
A. georgica, ya que esta especie de pato es de comportamiento gregario y sociable con otras especies de aves acuáticas durante los meses invernales (Kolbe, 1999). Por otro lado, se ha sostenido que existiría una estrecha relación entre el tamaño corporal del Phthiraptera y el de su hospedador (Tompkins \& Clayton, 1999), lo que podría actuar como una barrera para el establecimiento de poblaciones viables en hospedadores potenciales. Esto explicaría que las tres especies de Phthiraptera solo se registren en especies de una misma familia, ya que serían similares en tamaño, fisiología y conducta.

En relación a los endoparásitos, en general poseen ciclos de vida indirectos, es decir, necesitan un hospedador definitivo y uno o más hospedadores intermediarios para poder completar su ontogenia. Sin embargo, una diferencia importante entre los ciclos de vida de Digenea y Cestoda, es que los primeros se multiplican asexualmente en su primer hospedador intermediario, lo que puede llevar a una mayor abundancia en el hospedador definitivo, a diferencia de los Cestoda, donde solo pocas especies poseen esta característica (Poulin \& Mouillot, 2004).

En cuanto a la especificidad de los endoparásitos encontrados en el presente estudio, el estado adulto de $A$. burti solo se ha reportado en especies de la familia Anatidae. $\mathrm{Su}$ estado larval se ha registrado en Lymnaea stagnalis (Linnaeus, 1758) y Radix auricularia (Linnaeus, 1758) (Gastropoda: Pulmonata) (Faltynkova \& Haas, 2006), especies de moluscos no registradas hasta ahora en Chile, lo que no descarta su presencia, debido al escaso conocimiento estos taxa en el país (Valdovinos, 2006). Por su parte, $P$. pseudoalveatum tuvo una baja prevalencia y alta intensidad, lo que puede deberse a la característica de algunos macroparásitos de agruparse fuertemente en pocos individuos por razones de variaciones en la susceptibilidad de los individuos o la naturaleza contagiosa de las infecciones (Clayton et al., 2003). No hay estudios respecto a los hospedadores intermediarios para esta especie. $N$. imbricatus ha sido encontrado en diferentes aves y mamíferos, lo que explica el alto valor del índice de STD (lo que indica una baja especificidad), hecho que concuerda con la menor prevalencia de los tres Digenea. Se han 
registrado moluscos gastrópodos de las familias Bithyniidae, Planorbidae y Lymnaeidae como hospedadores intermediarios (Ataev et al., 2002), sin existir precisión sobre las especies que estarían participando en su ciclo vital en Chile. En cuanto a los cestodos, $F$. propeteres (Fuhrmann, 1907) Spassky, 1966 (Syn. Lateriporus propeteres Fuhrmann, 1907) y $C$. megalops (Nitzsch in Creplin, 1829) Wolffhügel, 1938 (Syn. Hymenolepis megalops Nitzsch in Creplin, 1829) son especies monotípicas. Esta última especie posee una amplia distribución geográfica y se ubica en el ano y cloaca de las aves Anseriformes y raramente en Galliformes y Gruiformes (Muniz-Pereira \& Amato, 1998). Entre sus hospedadores intermediarios se ha descrito a los ostrácodos Cypris pubera Müller, 1776 (Haukos \& Neaville, 2003), Eucrypis inflata Sars, 1903 y Heterocypris incongruens (Ramdohr, 1808) (Ostracoda: Cypridae). Solo $H$. incongruens ha sido descrito en Chile (Berríos \& Sielfeld, 2000), por lo que se asume que actúa como hospedador intermediario.

Poulin (1996) describe que las diferencias entre la abundancia y riqueza de los parásitos y el sexo del hospedador se deben a factores morfológicos, como el tamaño relativo del hospedador, factores fisiológicos como diferencias en los niveles hormonales por estrés, inmunosupresión o condiciones reproductivas, y a factores etológicos como comportamiento, interacciones sociales y alimentación. El hecho de que las aves del presente estudio fueran capturadas durante un período de tiempo no superior a los tres meses, podría explicar la similitud en condiciones fisiológicas, etológicas y niveles hormonales de los patos, lo que estaría influyendo en que no existan diferencias significativas en la abundancia y riqueza de los parásitos entre sexos de las aves hospedadoras.

La mayor abundancia y riqueza de parásitos en algunas localidades puede deberse a que a su vez existe una mayor abundancia o riqueza de los hospedadores intermediarios en aquellos lugares. La no caracterización de los lugares de muestreos, así como el desconocimiento de los hospedadores intermediarios, impiden hacer mayores inferencias al respecto. Más aún, el único parásito que mostró diferencias en abundancia entre localidades fue $P$. pseudoalveatum, parásito cuyo ciclo ha sido escasamente estudiado.
Respecto a la dieta de A. georgica en Chile, Schlatter et al. (1983) afirman que es una especie generalista que incluye en su dieta un alto porcentaje de semillas y frutas, con una alta similitud entre edades y sexos, lo que sumado a la poca información referida a la biodiversidad de invertebrados de los sistemas límnicos en Chile, hace difícil especular qué invertebrados formarían parte del ciclo de los parásitos del presente trabajo. Por otro lado, la mayoría de las aves capturadas, no contenían alimento en su estómago y las que lo poseían, solo presentaban semillas, algas u hojas, por lo que no se pudo sugerir posibles hospedadores intermediarios.

Finalmente, cabe destacar que los Phthiraptera Anaticola crassicornis y Trinoton querquedulae (Linneus, 1758), y los endoparásitos Nematoda Porrocaecum sp. y los Cestoda Cloacotaenia megalops y Fuhrmanacanthus propeteres constituyen primeros registros para Chile.

\section{AGRADECIMIENTOS}

Este estudio forma parte de la tesis de magíster en zoología de la primera autora, realizado en la Universidad de Concepción. Nuestros agradecimientos a Armando Cicchino, Lya Lunaschi y Boyko Gergiov por su valiosa colaboración en la determinación taxonómica de los parásitos.

\section{LITERATURA CITADA}

ANDERSON R, A CHABAUD \& S WILLMOTT (eds) (1974) CIH Keys to the Nematode Parasites of Vertebrates ( $\left.{ }^{\text {os }} 1-10\right)$. Commonweath Agricultural Bureaux, Farnham Royal, Buck, UK.

ATAEV G, E KOZMINSKII \& A DOBROVOLSKII (2002) Dynamics of infection of Bithynia tentaculata (Gastropoda: Prosobranchia) with trematodes. Parazitologiia 36: 203-218.

BERRÍOS V \& W SIELFELD (2000) Superclase Crustacea, guías de identificación y biodiversidad fauna chilena. Apuntes de zoología, Universidad Arturo Prat. Iquique, Chile. $32 \mathrm{pp}$

BUSH A, K LAFFERTY, J LOTZ \& A SHOSTAK (1997) Parasitology meets ecology on its own terms: Margolis et al. revisited. Journal of Parasitology 83: 575-583.

CATTAN P \& I TAGLE (1974) Estudio preliminar de la helmintiasis gastrointestinal en el conejo silvestre Oryctolagus cuniculus. Revista de la Sociedad de Medicina Veterinaria (Chile) 24: 34-42

CICCHINO A \& D DEL CASTRO (1998) Ichnocera. En: 
Morrone J \& S Coscaron (eds) Biodiversidad de artrópodos argentinos: 104-124. Ediciones Sur, La Plata, Argentina.

CLARKE K \& R WARWICK (1999) The taxonomic distinctness measure of biodiversity weighting of step lengths between hierarchical levels. Marine Ecology Progress Series 184: 21-29.

CLARKE K \& R WARWICK (2001) A further biodiversity index applicable to species lists: variation in taxonomic distinctness. Marine Ecology Progress Series 216: 265-278

CLAY T (1963) New species of Trinoton Nitzsch (Mallophaga, Insecta). Memoirs of the Queensland Museum 14: 87-97.

CLAYTON D, S AL-TAMINI \& K JONSON (2003) The ecological basis of coevolutionary history. En: Page RDM (ed) Tangled trees: Phylogeny, cospeciation and evolution: 310-341. University of Chicago Press, Chicago.

DEL HOYO J, A ELliOTT, J SARGATAL (1992) Handbook of the birds of the World Vol. 1 (Ostrich to Ducks). Linx Edicions, Barcelona, España: 536-638.

DRAGO F, L LUSNACHI, A HINOJOSA-SÁEZ \& D GONZÁLEZ-ACUÑA (2007) First record of Australapatemon burti and Paramonostomun pseudoalveatum (Digenea) from Anas georgica (Aves: Anseriformes) in Chile. Acta Parasitologica 52: 201-205.

EICHLER W \& T VASJUKOVA (1980) Die Mallophagengattung Anaticola (Phthiraptera: Mallophaga). Deutsche Entomologische Zeitschrift 27: 335-375

FALTYNKOVA A \& W HAAS (2006) Larval trematodes in freshwater molluscs from the Elbe to Danube rivers (Southeast Germany): before and today. Parasitology Research 99: 1572-1582.

FIGUEROA R, J CERDA \& C TALA (2001) Guía de aves dulceacuícolas de Aysén. Servicio Agrícola y Ganadero, Región de Aysén, Ministerio de Agricultura, Gobierno de Chile. 184 pp.

GONZÁLEZ D, O SKEWES, C CANDIA, R PALMA \& L MORENO (2005) Estudio del parasitismo gastrointestinal y externo en Caiquén Chloephaga picta Gmelin 1789 (Aves, Anatidae) en la Región de Magallanes, Chile. Parasitología Latinoamericana 60: 86-89.

HAFNER MS, PD SUDMAN, FX VILLABLANCA, TA SPRADLING, JW DEMASTES, \& SA NADLER (1994) Disparate rates of molecular evolution in cospeciating hosts and parasites. Science 265: 1087-1090.

HAUKOS D \& J NEAVILLE (2003) Spatial and temporal changes in prevalence of a cloacal cestode in wintering waterfowl along the Gulf coast of Texas. Journal of Wildlife Diseases 39: 152-160.

HOUSSE RE (1945) Las aves de Chile en su clasificación moderna. Su vida y costumbres. Ediciones Universidad de Chile, Santiago, Chile. 390 pp.

KHALIL L \& A JONES (1994) Keys to the cestode parasites of vertebrates. Department of Zoology. The Natural History Museum, London. Cambridge, UK. 747 pp.

KÉLER S (1957) Uber die deszendenz und die differenzierung der Mallophagen. Parasitology Research 18: 55-160

KÉLER S (1960) Uber die dualistische differenzierung der gattung Anatoecus cummings (Mallophaga). Parasitology Research 20: 207-316.

KOLBE H (1999) Die entenvogel der welt. Verlag Ulmer. Stuttgart, Germany. $376 \mathrm{pp}$
KRASNOV B, G SHENBROT, I KHOKHLOVA \& R POULIN (2004) Relationship between abundance and the taxonomic distance among a parasite's host species: An example with fleas parasitic on small mammals. International Journal for Parasitology 34: 1289-1297.

LUQUE J, D MOUILLOT \& R POULIN (2004) Parasite biodiversity and its determinant in coastal marine teleost fishes of Brazil. Parasitology 128: 671-682.

MADGE S \& H BURN (2000) Wassergeflügel, ein bestimmungsbuch der schwäne, gänse und enten der welt. Berlin, Germany. 297 pp.

MARTÍNEZ D \& G GONZÁLEZ (2004) Las Aves de Chile, nueva guía de campo. Ediciones del Naturalista, Santiago, Chile. 620 pp.

MARSHALL A (1981) The ecology of ectoparasitic insects. Academic Press, New York, USA. 459 pp.

MUNIZ-PEREIRA L \& S AMATO (1998) Fimbriaria fasciolaris and Cloacotaenia megalops (Eucestoda, Hymenolepididae), Cestodes from Brazilian Waterfowl. Memorias del Instituto Oswaldo Cruz 93: 767-772.

OLMOS V \& M GEORGE-NASCIMENTO (1997) El gremio de larvas de Digenea en el caracol del sur de Chile Chilina dombeyana: ¿qué indica la tasa metabólica de los hospedadores parasitados? Revista Chilena de Historia Natural 70: 109-118.

PALMA R (1978) Slide-mounting of lice: A detailed description of the Canada balsam technique. New Zealand Entomology 6: 432-436.

POULIN R (1996) Sexual inequalities in helminth infections: A cost of being a male? American Naturalist 147: 237-295.

POULIN R (1998) Evolutionary ecology of parasites: From individuals to communities. Chapman \& Hall, London, UK. 212 pp.

POULIN R (1999) The intra- and interspecific relationships between abundance and distribution in helminth parasites of birds. Journal of Animal Ecology 68: 719-725.

POULIN R \& D MOUILLOT (2003) Parasite specialization from a phylogenetic perspective: A new index of host specificity. Parasitology 126: 473-480.

POULIN R \& D MOUILLOT (2004) The relationship between specialization and local abundance: The case of helminth parasites of birds. Oecologia 140: 372-378.

POULIN R \& D KEENEY (2008) Host specificity under molecular and experimental scrutiny. Trends in Parasitology 24: 24-28.

PRICE R \& J BEER (1963) The Kurodaia (Mallophaga: Menoponidae) parasitic on the Strigiformes, with a key to the species of the genus. Annal Entomological Society 55: 849-857.

PRICE R, R HELLENTHAL, R PALMA, K JOHNSON \& D CLAYTON (2003) The chewing lice: World checklist and biological overview. Illinois, USA. $501 \mathrm{pp}$.

SCHLATTER R, D ALDRIDGE, M ROMERO \& M HOFMANN (1983) Ecological studies of Chilean ducks. En: Boyd EH (ed) First western hemisphere waterfowl and waterbird Symposium: 133-137. Special Publication. Canadian Wildlife Service for the International Waterfoul Research Bureau, Edmonton, Canada.

SCHLATTER R, J SALAZAR, A VILLA \& J MEZA (1991) Reproductive biology of black-necked swans Cygnus melancoryphus at three Chilean wetland areas and feeding ecology at Río Cruces. Wildfowl Supplement 1: 268-271. 
SCHMIDT GD (1970) How to know the Tapeworms. Brown Company Publishers, Dubuque, Iowa, USA. $266 \mathrm{pp}$.

SCHMIDT G \& L ROBERTS (1984) Fundamentos de Parasitología. Principios y Conceptos Básicos. Editorial Continental, México. 655 pp.

SAG (2001) Cartilla para cazadores. Departamento de Protección de Recursos Naturales Renovables, Ministerio de Agricultura, Chile. 99 pp.

SIMKOVÁ A, D KADLEC \& M GELNAR (2002) Abundance-prevalence relationship of gill congeneric ectoparasites: Testing the core satellite hypothesis and ecological specialisation. Parasitology Research 88: 682-686.

SKRJABIN K (1964) Keys to the trematodes of animal and man. University of Illinois, Urbana, USA. 350 pp.

TENDEIRO J \& L MENDES (1994) Etudes sur les Colpocephalum (Mallophaga, Menoponidae) parasites des Falconiformes. III Quelques observations sur le "groupe polybori" Price \& Beer, avec description de trois espéces nouvelles. Garcia de Orta, Série de Zoología. Lisboa 20: 137-14.
TOMPKINS L \& D CLAYTON (1999) Host resources govern the specificity of swiftlet lice: Size matters. Journal of Animal Ecology 68: 489-500.

TORRES P \& C JARA (1986) Aegla denticulata (Crustacea: Decapoda): un nuevo huésped para Hysterothylacium sp. (Nematoda: Anisakidae) en el sur de Chile. Parasitología al Día 10: 134-135.

VALDOVINOS C (2006) Estado del conocimiento de gastrópodos dulceacuícolas de Chile. Gayana 70: 88-95.

YAMAGUTI S (1958) Systema Helminthum. The Digenetic trematodes of vertebrates. Vol. I. Interscience Publishers, London, UK. 1573 pp.

YAMAGUTI S (1959) Systema Helminthum. The cestodes of vertebrates. Vol II. Interscience Publishers, London, UK. $860 \mathrm{pp}$

YAMAGUTI S (1961) Systema Helminthum. The nematodes of vertebrates. Vol III. Interscience Publishers, London, UK. 1261 pp.

ZAR J (1984) Biostatistical Analysis. Second Edition. Prentice Hall Inc, Englewood Cliffs, New York, USA. 718 pp. 
